\section{PP.03 DOES THE APGAR SCORE MATTER? INVESTIGATING THE RELATIONSHIP BETWEEN A LOW SCORE AND ADVERSE OUTCOMES FROM BIRTH TO CHILDHOOD}

doi:10.1136/archdischild-2013-303966.285

${ }^{2} \mathrm{GL}$ Malin, ${ }^{1} \mathrm{RK}$ Morris, ${ }^{4} \mathrm{~S}$ Ahmad, 'R Riley, ${ }^{3} \mathrm{KS}$ Khan. 'University of Birmingham, Birmingham, UK; ${ }^{2}$ University of Nottingham, Nottingham, UK; ${ }^{3}$ The Blizard Institute, Barts and the London School of Medicine, London, UK; ${ }^{4}$ The Royal Derby Hospital, Derby, UK

Background A low Apgar score at birth may occur for a variety of reasons. Existing evidence regarding the long term implications is conflicting.

Methods Systematic review of the literature, with random effects meta-analysis.

Results 87 manuscripts were included, with a total of $3,690,080$ neonates. A low Apgar score was strongly associated with neonatal mortality in a population born at term ( $\geq 37$ weeks gestation), or with normal birth weight $(\geq 2.5 \mathrm{~kg})(10$ minute Apgar score $\leq 3:$ OR 1417.75, 95\% CI 915.99 to 2194.36). Raising the Apgar score at a particular time reduced the strength of association. In a pre-term population, the association was smaller (10 minute score $\leq 3$ : OR $66.49,95 \%$ CI 45.00 to 98.22 ). For neonatal morbidity, significant association was seen at a number of thresholds. In a term population, there was a significant association between a low Apgar score and cerebral palsy at all thresholds examined, with the largest association seen at a 5 minute Apgar score $\leq 3$ (3 studies, OR 46.35, 95\% CI 11.21 to 191.59). When the predictive ability of a low Apgar score was considered, the specificity and positive likelihood ratios were generally high, however the corresponding sensitivity and negative likelihood ratios were low.

Conclusion A low Apgar score at birth is strongly associated with neonatal mortality, morbidity and childhood cerebral palsy, particularly in a term or normal birth weight population. Further research is required to identify the threshold at which the Apgar score may best predict adverse outcomes.

\section{PP.04 ANTENATAL CORTICOSTEROIDS, FOR WOMEN WITH DIABETES, UNDERGOING ELECTIVE LOWER UTERINE SEGMENT CAESAREAN SECTION BETWEEN $38+0-38+6$, ARE THEY WORTH IT?}

doi:10.1136/archdischild-2013-303966.286

K Hodson, C Lyon-Dean, S Marshall, M MacDougall. Royal Victoria Infirmary, Newcastle upon Tyne, UK

Background The Royal College of Obstetricians and Gynaecologists (RCOG) recently recommended antenatal corticosteroids for all women undergoing elective lower uterine segment caesarean section (El-LSCS) prior to $38^{+6}$ weeks. National Institute for Health and Clinical Excellence recommend delivery for women with diabetes at $38^{+0}$ weeks. Corticosteroids can destabilise maternal glycaemic control. The purpose of this study was to assess the outcomes in women with diabetes undergoing El-LSCS between $37^{+6}$ and $38^{+6}$ weeks who did not receive antenatal corticosteroids prior to the RCOG guideline.

Methods We performed a retrospective audit of 27,869 consecutive live births in a tertiary referral hospital. We calculated admission rates to Special Care Baby Unit (SCBU) in those undergoing El-LSCS without corticosteroids, identifying presence of diabetes, type of diabetes and gestation at delivery.

Results $32 \%(\mathrm{n}=985 / 3016)$ of El-LSCS were performed prior to $38^{+6}$ weeks in the general population. In the diabetic population $70 \%\left(\mathrm{n}=51 / 72\right.$ ) of El-LSCS were performed prior to $38^{+6}$ weeks. $41.6 \%$ ( $n=30 / 72$ ) of which were performed between $38^{+0}-38^{+6}$ weeks.

The admission rate to SCBU in the diabetic population undergoing El-LSCS was $1.38 \%$, lower than both the $4.7 \%$ rate in the general population undergoing El-LSCS and the $2.7 \%$ overall rate in the diabetic population. No women with diabetes undergoing El.LSCS after $37^{+6}$ weeks had a baby admitted to SCBU despite not receiving corticosteroids.

Conclusion The admission rates to SCBU observed in babies born to mums with diabetes delivering between $38^{+0}-38^{+6}$ by El-LSCS without corticosteroid cover, does not support their routine administration in this population, especially given the destabilising effect they have on glycaemic control.

\section{PP.05 WITHDRAWN BY AUTHOR}

\section{PP.06 UK OBSTETRIC CRITICAL CARE PROVISION REMAINS UNFIT FOR PURPOSE}

doi:10.1136/archdischild-2013-303966.287

${ }^{1}$ A Saunders, ${ }^{1 F}$ Jones, ${ }^{4} \mathrm{~A}$ Carlin, ${ }^{3} \mathrm{H}$ Scholefield, ${ }^{2,5} \mathrm{MK}$ Whitworth. 'University of Manchester, Manchester, UK; '2St Mary's Hospital CMFT, Manchester, UK; '3iverpool Women's Hospital, Liverpool, UK; 'John Hunter Hospital, Newcastle, Australia; ${ }^{5}$ Maternal \& Fetal Health Research Centre, University of Manchester, Manchester, UK

Background Successive confidential enquiries have recommended that obstetric critical care (OCC) patients be cared for in a level 2 setting with adequate facilities and trained staff. In 2007 we conducted the first national survey of UK OCC provision and demonstrated major, potentially life threatening deficiencies. We aim to see if OCC provision in 2012 is fit for purpose.

Method We conducted a validated survey of 227 maternity units in the UK (May-July 2012).

Results 137 questionnaires were returned (response rate-60\%). Mean number of deliveries/year was 4076 (200-9867). 57\% of units report having designated OCC bed provision ( $56 \%$ in $2007 \mathrm{p}=0.88$ ). Median provision is two beds/unit compared with one in 2007. Nursing care is provided solely by midwives in $71 \%$ of units (95\% in 2007 , $\mathrm{p}<0.05) .76 \%$ of these midwives have some formal in-house or external OCC training (33\% in 2007, $\mathrm{p}<0.05$ ). Joint medical care is provided by obstetricians and anaesthetists in $89 \%$ of units $(72 \%$ in 2007 , $\mathrm{p}<0.05)$. However, of those units claiming to have designated OCC capacity $32 \%$ were unable to provide one or more of the elements required for level 2 care e.g. arterial line management. The main issues identified by responding units remain the need for; better facilities and equipment, formal OCC training, improved staffing.

Conclusions Our survey suggests there is a lack of knowledge about OCC and provision remains unfit for purpose. We remain a long way from providing equity of critical care for pregnant women as recommended by the joint RCOG/RCA document of 2011.

\section{PP.07 EARLY FETAL LOSS IN MONOCHORIONIC AND DICHORIONIC TWIN PREGNANCIES: THE STORK MULTIPLE PREGNANCY COHORT}

doi:10.1136/archdischild-2013-303966.288

F D'Antonio, A Khalil, T Dias, A Bhide, B Thilaganathan. Fetal Medicine Unit, Division of Developmental Sciences, ST. George's Univeristy of London, London, UK

Objectives The aim of this study is to compare the early perinatal loss rates between $\mathrm{MC}$ and dichorionic (DC) twins in an era of invasive treatment for TTTS

Methods This was a retrospective study of all twin pregnancies of known chorionicity from a large regional cohort of 9 hospitals over a ten year period. Ultrasound data were matched to hospital delivery records and a mandatory national register of pregnancy losses. Prospective risk of pregnancy loss from 14 to 24 weeks' gestation was calculated and the survival trend of $\mathrm{MC}$ and $\mathrm{DC}$ twins was analysed using Kaplan-Meier survival analysis. 